\title{
Archéopages
}

Archéopages

Archéologie et société

\section{Le trophée de Décines en territoire allobroge. Un témoignage des pratiques guerrières gauloises}

\section{Emmanuel Ferber}

\section{(2) OpenEdition}

1 Journals

Édition électronique

URL : https://journals.openedition.org/archeopages/534

DOI : 10.4000/archeopages.534

ISSN : 2269-9872

\section{Éditeur}

INRAP - Institut national de recherches archéologiques préventives

\section{Édition imprimée}

Date de publication : 1 septembre 2014

Pagination : 16-21

ISSN : 1622-8545

\section{Référence électronique}

Emmanuel Ferber, «Le trophée de Décines en territoire allobroge. Un témoignage des pratiques guerrières gauloises », Archéopages [En ligne], 39 | 10/2013-01/2014, mis en ligne le 01 janvier 2016, consulté le 21 janvier 2022. URL : http://journals.openedition.org/archeopages/534 ; DOl : https:// doi.org/10.4000/archeopages.534 


\section{Le trophée de Décines en territoire allobroge Un témoignage des pratiques guerrières gauloises}

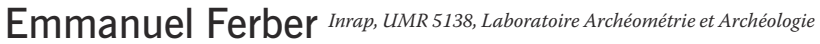

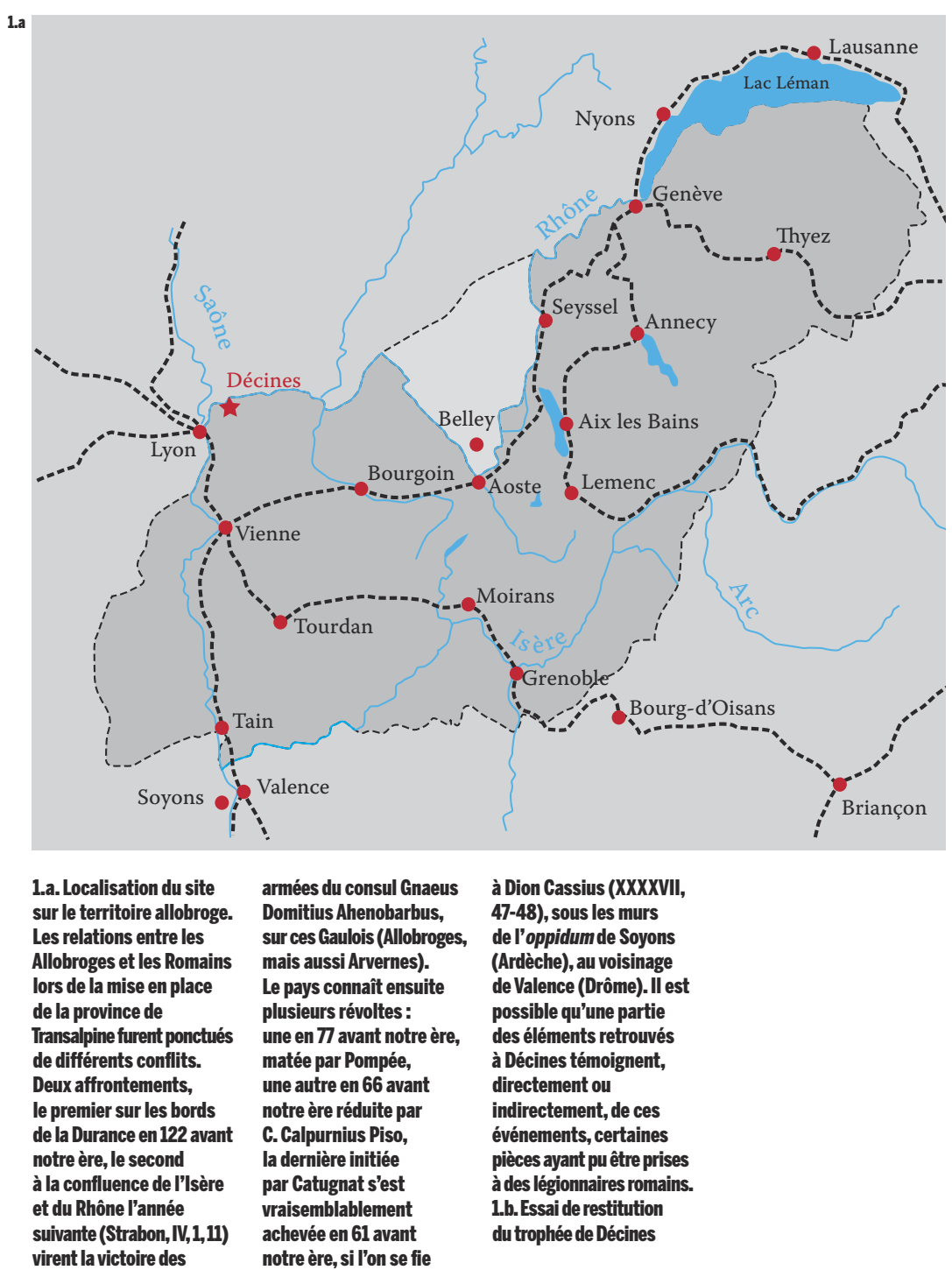

i

Territoire allobroge

Territoire statut incertain

$\star$ Sanctuaires de Décines

---- Route

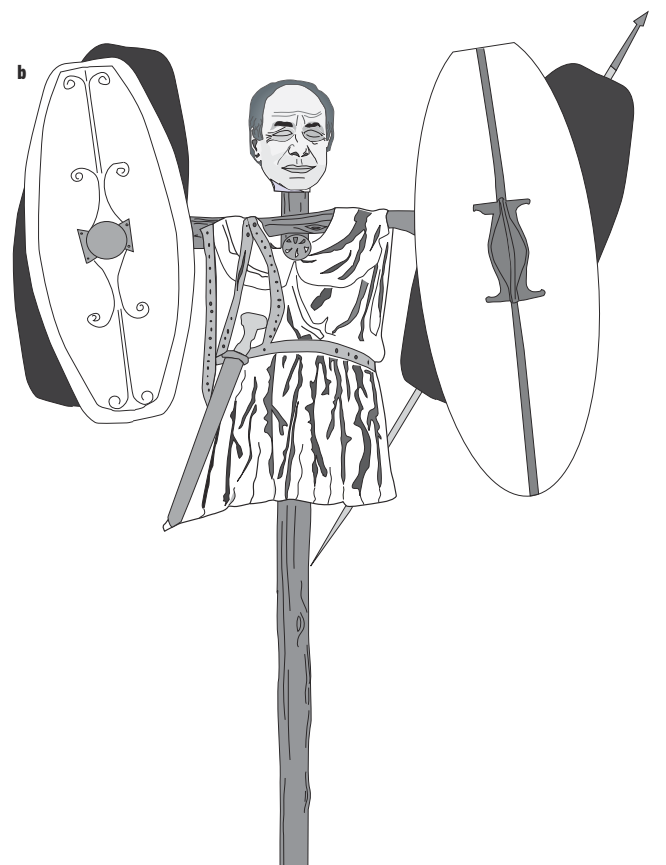


La fouille menée en 2011 à Décines-Charpieu (Rhône) ${ }^{\mathbf{1}}$ a mis en évidence des occupations s'étendant du Néolithique ancien au $\mathrm{XVI}^{\mathrm{e}}$ siècle (Ferber, Plantevin, 2013 ; Ferber et al., 2012). Un enclos fossoyé, daté de La Tène $\mathrm{D}_{1}^{2}$ (fin $\mathrm{du} \mathrm{II}^{\mathrm{e}}$ siècle-première moitié du $\mathrm{I}^{\mathrm{er}}$ siècle avant notre ère), a été mis au jour dans cet espace situé sur la bordure nord-ouest du territoire allobroge, qui s'étendait des Alpes jusqu'au Rhône et à l'Isère [ill. 1]. Le mobilier découvert comprend des fragments de crânes humains et des pièces d'armement qui ont été interprétés comme les vestiges d'un trophée.

\section{Les fragments de crânes et le mobilier découverts}

L'enclos, de plan carré ou rectangulaire, se développe en partie hors de l'emprise de la fouille, ce qui a réduit les possibilités d'en définir la fonction de façon assurée. À l'intérieur de cet ensemble, l'aire étudiée n'a révélé qu'une petite structure fossoyée localisée sur sa bordure méridionale [ill. 2]. Les fragments de torchis, la céramique, et les restes de galettes calcinées ${ }^{3}$ retrouvés à proximité plaident en faveur d'une zone d'habitat.

Les strates de comblement du fossé se sont révélées relativement pauvres en mobilier à l'exception de celles occupant son angle sud-est. Dans ce secteur, les restes de deux voire trois crânes humains ${ }^{4}$ ont été retrouvés. Le premier a appartenu à un individu d'âge mûr ; il présente une entaille sur l'occipital caractéristique d'une probable tentative de décapitation [ill. 3]. Du deuxième, il ne subsiste que la partie arrière de la boîte crânienne qui a appartenu à un grand adolescent ou à un jeune adulte. Il porte les traces nettes d'un sciage régulier, transversal, partant verticalement du sommet des pariétaux sur environ la moitié du pariétal gauche. L'os semble ensuite cassé jusqu'à l'occipital [ill. 4]. Le troisième fragment est un morceau de maxillaire qui appartient lui aussi à un grand adolescent. Il est possible que les deux derniers éléments correspondent au même individu.

Par ailleurs, on recense plusieurs pièces d'armement : quatre umbones (coque métallique protégeant la main) de bouclier, une pointe de pilum ou de javeline et un glaive. Si trois des umbones sont des modèles à ailettes rectangulaires ou trapézoïdales encadrant une coque hémisphérique typiques de La Tène $\mathrm{D} 1$, le dernier [ill. 5] présente une coque bivalve fuselée entourée d'un replat qui s'évase aux deux extrémités. Deux rivets maintiennent les deux parties assemblées sur la spina, mais aucune trace de fixation (rivet ou trace de rivet) n'a été observée permettant d'assurer le maintien de la pièce sur le plat du bouclier lui-même. Sa forme générale évoque certains modèles de l'armée tardo-républicaine (Poux, 2008, p. 410). Cette pièce peut être associée au glaive, arme emblématique du légionnaire, caractérisée par une lame courte et légèrement cintrée [ill. 6]. La pointe pyramidale sur douille [ill. 7], quant à elle, est comparable à des exemples retrouvés à Alésia (52 avant notre ère) ou à Valentia (7o avant notre ère). À cet ensemble s'ajoutent deux ustensiles domestiques en fer : une pelle à feu au manche torsadé et une louche munie d'une poignée horizontale de o,40 m [ill. 8].

Ces objets, liés pour la plupart au domaine militaire, pourraient être les vestiges disloqués d'un trophée guerrier surplombant l'angle sud-est de l'enclos. Ce type de monument, connu dans le monde celte par les textes (Tite-Live, Histoire romaine, VII, 39,1) peut prendre diverses formes illustrées par des observations de terrain.

\section{Quelques éléments de comparaison}

À Ribemont-sur-Ancre (Somme) ${ }^{\mathbf{5}}$, un enclos fossoyé daté du $\mathrm{III}^{\mathrm{e}}$ siècle avant notre ère conservait les dépouilles d'individus dont les os ont connu des manipulations méticuleuses. Juste à l'extérieur de cette enceinte sacrée, cent quatorze guerriers acéphales et en armes, décédés pour la plupart de mort violente, ont été suspendus et exposés serrés les uns contre les autres (Rousseau, 2012). À Gournay-sur-Aronde (Oise) ${ }^{\mathbf{6}}$, les comblements d'un fossé d'enclos quadrangulaire daté des $\mathrm{III}^{\mathrm{e}}$ et $\mathrm{II}^{\mathrm{e}}$ siècles ont livré une multitude d'armes ainsi que quelques crânes. L'étude a permis de comprendre que le fossé avait été remblayé avec les rejets de structures proches du fossé et l'hypothèse d'une exposition de reliques a été émise. Le degré d'oxydation des pièces laisse deviner qu'elles sont restées exposées longtemps, probablement jusqu'à ce que la corrosion les fasse choir d'elles-mêmes (Brunaux, Rapin, 1988, p. 135). On peut aussi citer le dépôt découvert dans le sanctuaire de Tintignac (Corrèze) fréquenté au $\mathrm{I}^{\mathrm{er}}$ siècle avant notre ère. Au cœur d'un petit enclos fossoyé, une fosse votive recueille de nombreuses offrandes. Là se mêleraient des éléments de cérémonie (comme les fameux casques d'apparat) aux restes d'un trophée matérialisé par l'armement d'au moins neuf personnes (Maniquet, 2008, 2011).

Le trophée d'inspiration hellénistique, ce mannequin fait d'une croix de bois affublée des armes et autres objets pris à l'ennemi, tel qu'il est représenté sur nombres de revers de monnaies grecques puis républicaines, est aussi en usage en Gaule comme l'atteste un texte relativement tardif (Claude Elien, Histoire variée, XII, 23). Si l'iconographie sur le sujet est abondante (le thème du trophée perdure sur les monnaies et sur les arcs de triomphe durant tout l'Empire), les découvertes réelles, « en armes et en os », de ce type de réalisation demeurent rares. Le seul exemple comparable connu à ce jour a été découvert en 2009 au sanctuaire de Corent (Puy-de-Dôme) ${ }^{7}$. Daté de la fin du II $^{\mathrm{e}}$ siècle avant notre ère, il se compose d'une cotte de maille, d'une plaque de fourreau d'épée, de quatre umbones et des restes d'un sanglier porte-enseigne en bronze (Garcia,

\footnotetext{
1. Responsable d'opération :

2. Responsable de secteur :

Manon Cabanis, Inrap.

5. Fouille du Champ

crezette à Ribemont-

Brunaux.

6. Fouille du Parc à

(Oise), de 1975 à 198

responsable d'opération :

Jean-Louis Brunaux.

7. Fouille de Corent,

responsable d'opération:

Matthieu Poux.
} 


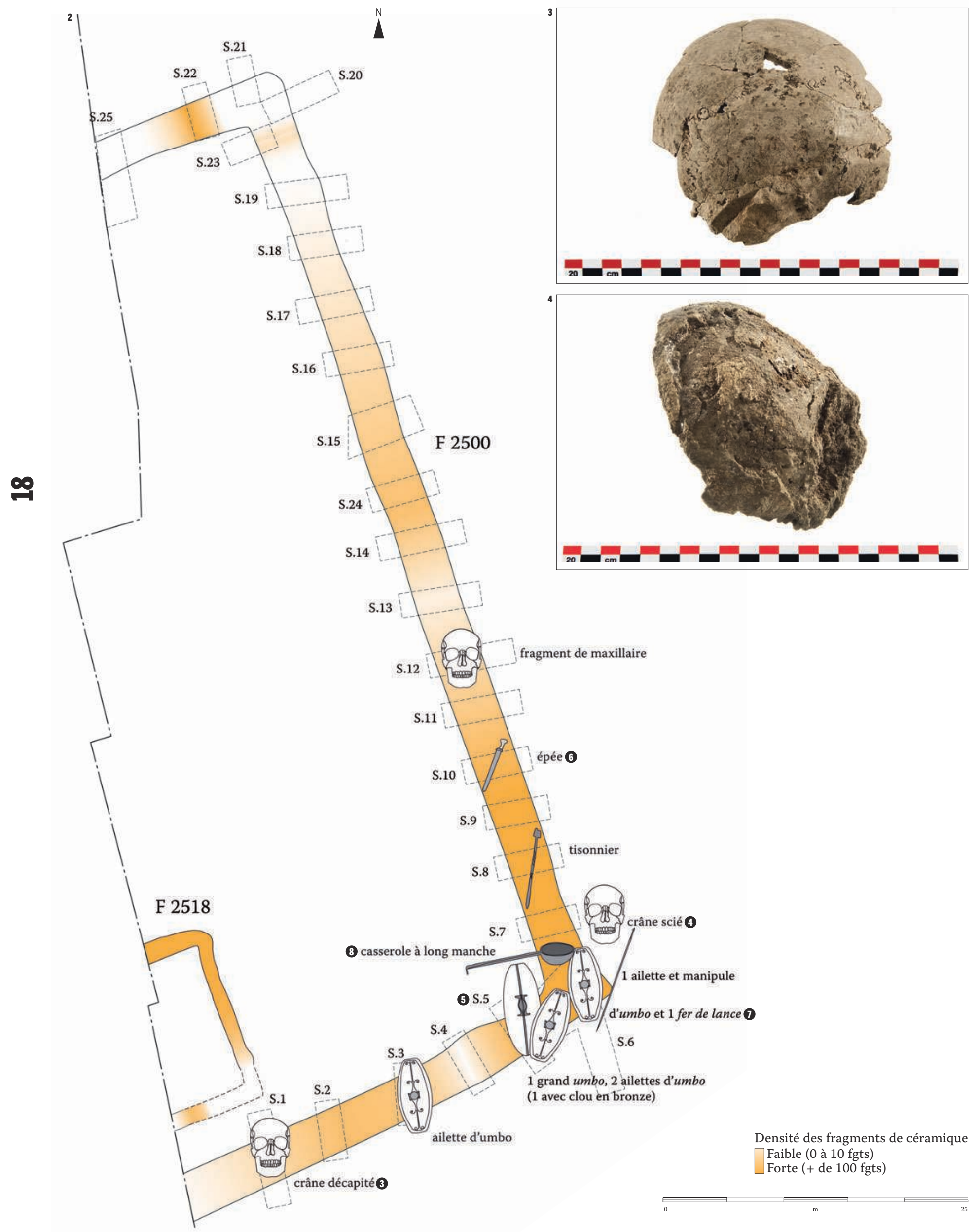


2. Répartition des objets métalliques et des

fragments humains

dans l'enclos fossoyé.

Le fossé oriental de l'enclos

fossoyé, long de $80 \mathrm{~m}$,

présente à l'ouverture

une largeur de 3,5 m

pour une profondeur

conservée de $1,5 \mathrm{~m}$.

Les terres décaissées ont

été placées sur la bordure

interne de la structure,

formant ainsi un talus

protecteur. L'existence

d'une palissade

couronnant l'ensemble

est envisageable, mais

ne peut être

formellement démontrée

3. Trace de décapitation

sur un crâne.

4. Fragment de crâne

humain scié.

5. Umbo de bouclier

tardo-republicain.

6. Glaive.

7. Pointe à douille.

8. Louche.
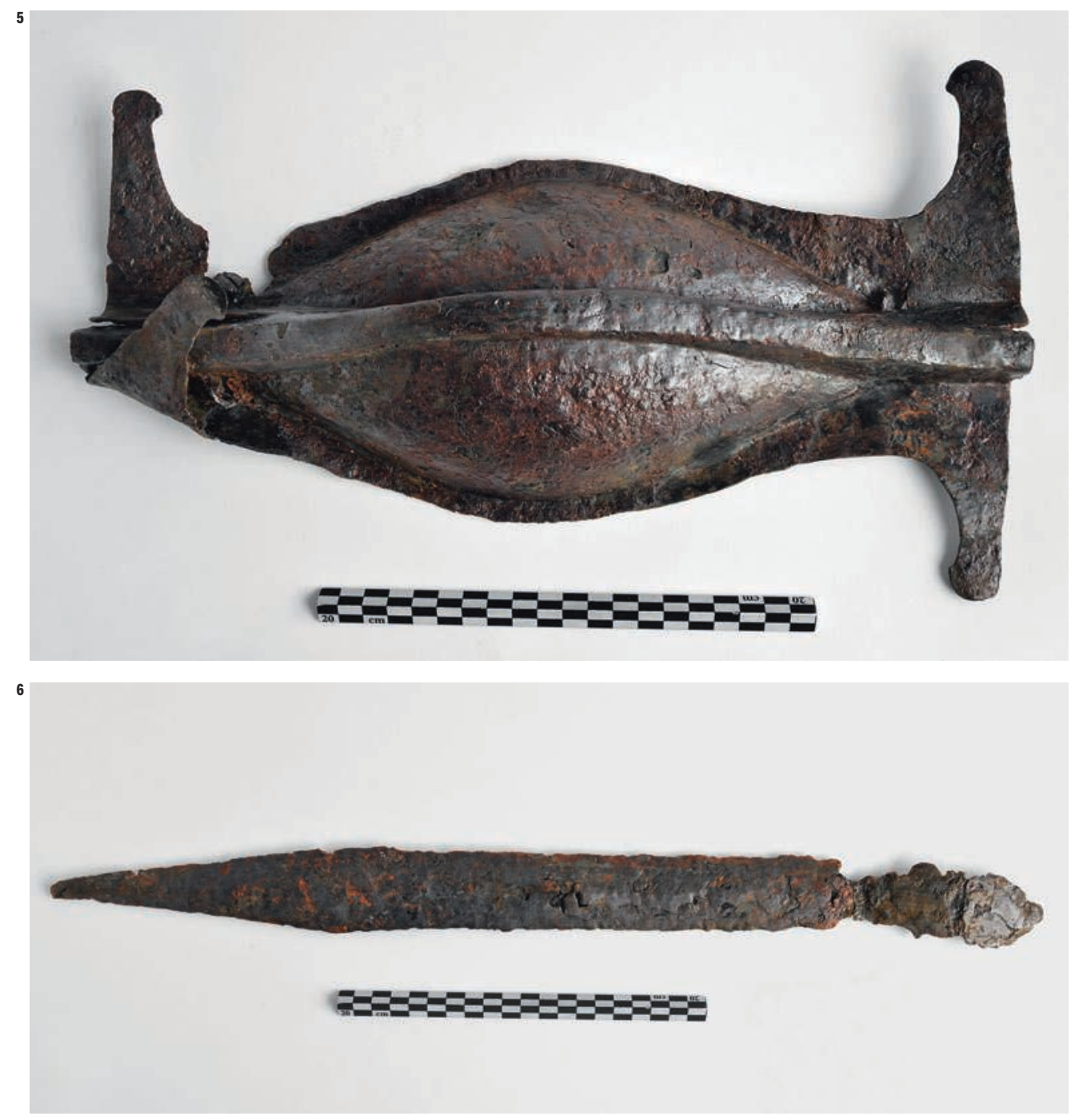

7
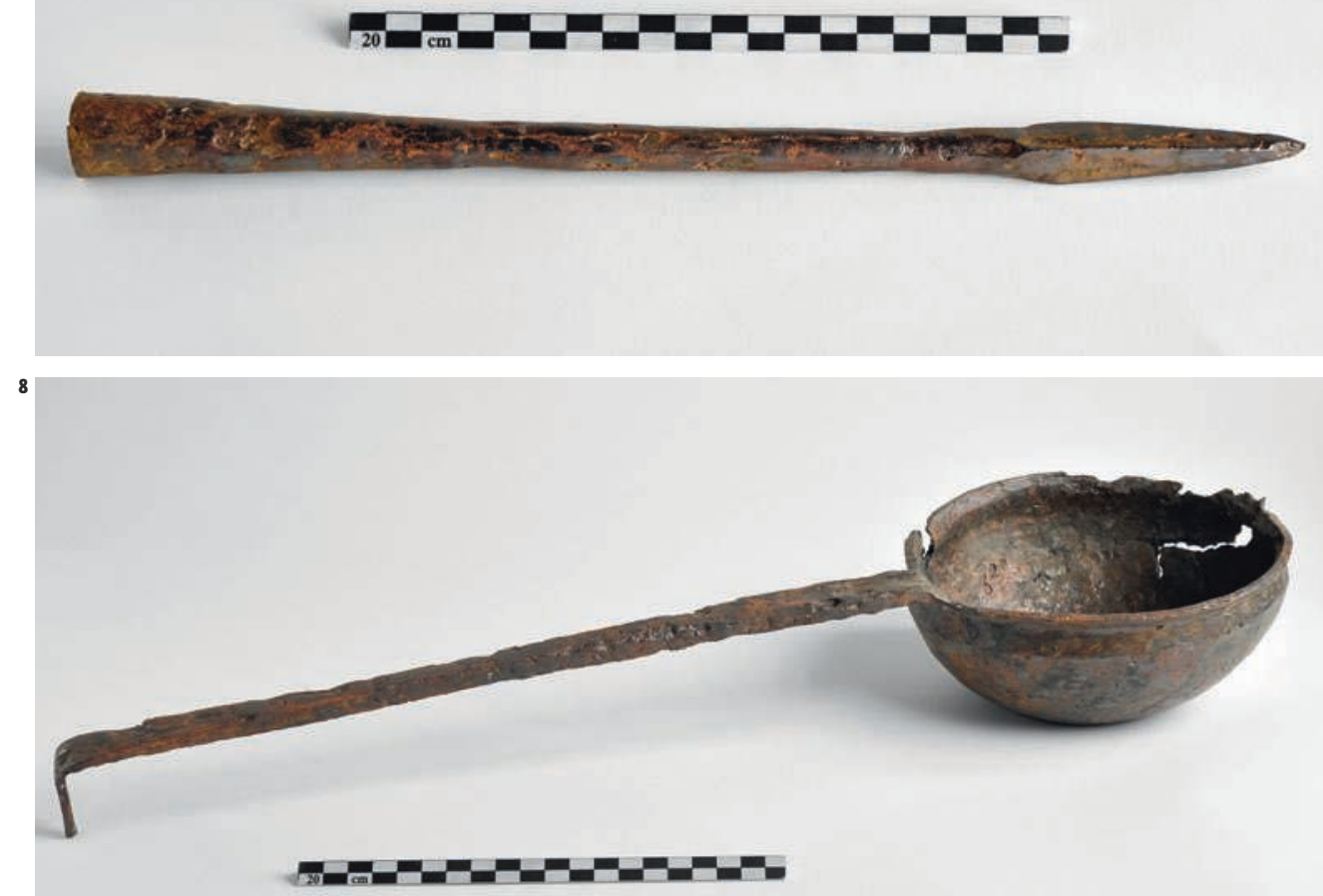
Demierre, 2011). Des fragments de crânes ont aussi été retrouvés à proximité immédiate de ces éléments (Guichon, Poux, 2011, p. 147). Sur les deux sites, à Corent comme à Décines, ont été découverts des umbones groupés permettant de supposer que les boucliers étaient fixés par paires, dos à dos, sur chacun des bras du mannequinguerrier. Si l'exemplaire de Décines semble plus modestement doté que celui de Corent, il se distingue par la présence de quelques pièces d'armement romain et par la mise en évidence de manipulations sur les crânes suggérant une exposition des têtes. En effet, il faut ici envisager l'exposition de têtes et non de crânes : si l'on se réfère aux textes et aux représentations figurées antiques (cf. infra), l'individu mis en exergue conserve généralement son apparence humaine.

\section{Les têtes coupées dans le monde antique}

Les têtes coupées jouent manifestement un rôle important dans la culture celtique. Certaines têtes conservées et exposées dans des loges céphaliques creusées dans des linteaux ont pu être celles des ancêtres ou des personnages les plus prestigieux de la communauté élevés ainsi au rang de héros. Il s'agirait alors de reliquaires (Arcelin, PlanaMallart, 2011). Cette hypothèse repose essentiellement sur des comparaisons ethnologiques, aucun texte ancien ne permettant d'en valider la pertinence.

En revanche, Poseidonios d'Apamée, auteur grec qui a visité les environs de Marseille vers 100 avant notre ère, soit à une date pratiquement contemporaine du trophée de Décines, évoque la pratique consistant à prélever la tête de son ennemi sur le champ de bataille. Dans un texte perdu mais dont des extraits ont été repris par Diodore de Sicile et Strabon, il raconte qu' « à l'issue des combat, les Gaulois suspendent au cou de leur chevaux les têtes de leurs ennemis et les ramènent chez eux pour les clouer à l'entrée des lieux de culte (Strabon, Géographie, IV, 4, 5) ou des habitats (Diodore de Sicile, Bibliothèque Historique, V, 29)». Les documents témoignant de cette coutume sont légion : outre une douzaine de textes recensés par Jean-Louis Brunaux (Brunaux, 2012, p. 109), les représentations iconographiques abondent. Parmi celles-ci, une gravure sur un vase trouvé à Aulnat (Puy-de-Dôme) et un bas-relief daté du $\mathrm{II}^{\mathrm{e}}$ siècle avant notre ère, taillé dans un bloc de pilier à Entremont (Bouches-du-Rhône) figurent explicitement la scène décrite en montrant des cavaliers portant à l'encolure de leurs chevaux des têtes (Ciesielski et al., 2011). On peut aussi penser à la statue du guerrier amassant devant lui une demi-douzaine de têtes trouvée sur l'oppidum d'Entremont (datée du $\mathrm{III}^{\mathrm{e}}$ siècle avant notre ère), ou encore à celle de l'homme assis sur un siège orné de têtes humaines exposée à Barcelone (datée $\mathrm{du} \mathrm{III}^{\mathrm{e}}-\mathrm{II}^{\mathrm{e}}$ siècle avant notre ère). Légèrement postérieures au trophée de Décines, des monnaies gauloises frappées à l'effigie de Dumnorix, chef éduen qui s'opposa à Jules César, le figure tenant dans sa main gauche, par les cheveux, une tête.

Des découvertes archéologiques témoignent de la réalité de ces pratiques, en particulier les corps acéphales de cent-quatorze guerriers du trophée de Ribemont-sur-Ancre (Brunaux, Rapin, 1988). Des crânes - dont certains sont sciés pour la réalisation de masques faciaux - ont été mis au jour au Cailar dans le Gard (associés par ailleurs à des armes, comme à Décines), dans un espace consacré daté du $\mathrm{III}^{\mathrm{e}}$ siècle avant notre ère (cf. article p.xx), ou à Corent dans le Puy-de-Dôme aux $\mathrm{II}^{\mathrm{e}}-\mathrm{I}^{\mathrm{e}}$ siècles avant notre ère (Rousseau, 2012, p. 123). Cette pratique a également été mise en évidence à une époque contemporaine de celle du site de Décines à Montmartin dans l'Oise (Rousseau, 2012, p. 123).

Toutefois, l'exposition de la tête de l'ennemi vaincu n'est pas l'apanage des seuls « barbares » : sur la colonne trajane, on ne compte pas moins de trois scènes de présentations de têtes de Daces à l'empereur (Depeyrot, 2008, p. 39, 115, 219) qui font par ailleurs pendant à l'exposition sur les hauts d'un rempart ennemi d'une demi-douzaine de têtes de romains empalées sur des pieux. Ces trophées accompagnés d'un vexillum représentent, semblet-il, les dépouilles du préfet Cornelius Fuscus et de ces hommes vaincus en 99 (Testart, 2012, p. 31).

Le nombre de crânes prélevés a sans nul doute un caractère ostentatoire et doit permettre de juger de la valeur du guerrier qui les a collectés, mais au-delà de cet aspect, on peut penser qu'en dépouillant ces adversaires de la partie du corps susceptible de faire l'objet d'un culte, on prive leur famille et leur clan des honneurs auxquels ils auraient pu prétendre.

Le trophée matérialiserait une victoire et semble être, à l'origine, mis en place sur le champ de bataille (Thucydide, dans son histoire de la guerre du Péloponnèse, décrit plusieurs fois ce rituel). L'effigie d'un guerrier vaincu, parée des armes, des habits, voire du crâne récupérés à l'issue des combats, pourrait symboliser l'ennemi donné en offrande aux dieux. Du lieu même du combat, le dépôt votif se serait ensuite déplacé sur le lieu de culte. Ainsi, Tite-Live (Histoire romaine, XXIII, 24) évoque la dépouille du général Lucius Postumius, vaincu par les Boiens en 216 avant notre ère, conduite dans un temple où sa tête, tranchée puis vidée, est décorée d'un cercle d'or pour servir de vase sacré. Si l'on en croit Plutarque (Alexandre-César XXVI-8) une épée prétendument subtilisée à Jules César trônait dans un sanctuaire arverne. Dans cette perspective, le trophée de Corent trouve naturellement sa place dans un édifice religieux. Cependant, comme nous l'avons déjà signalé, les prises de guerre peuvent aussi décorer l'habitat (Diodore de Sicile, Bibliothèque Historique, V, 29). En Allemagne (Bade-Wurtemberg), sur le site de Nordheim II, un ensemble de huit umbones de bouclier (qui, unis dos à dos comme à Corent, évoqueraient deux trophées) a été mis au jour dans un enclos fossoyé actuellement considéré comme un établissement rural (Nicolai, 2009). 


\section{Le trophée d'une victoire gauloise ?}

À Décines, les éléments découverts dans la partie fouillée de l'enclos fossoyé ne permettent pas de définir avec certitude le statut du site. Il en est de même du mobilier mis au jour dans les comblements du grand fossé. La faune est peu présente et mal conservée ( 42 fragments, 10 de cheval, 13 de bovin, 6 de capriné, 3 de porc). Si, en comparaison avec les autres secteurs du site, les fragments d'amphores - essentiellement des exemplaires de type Dressel 1A et des amphores gréco-italiques « récentes »- sont particulièrement concentrés dans cette structure, les 521 tessons ne permettent pas de caractériser un type d'activité lié à la consommation de vin. Le trophée pourrait trouver sa place aussi bien dans un sanctuaire que dans un enclos rural.

La présence, insolite au voisinage des armes et des fragments crâniens, d'ustensiles domestiques (une louche, un tisonnier) fait planer un doute sur la fonction du lot observé. Cet ensemble pourrait aussi évoquer les vestiges d'un habitat conservant les armes et les crânes d'ancêtres prestigieux. Bien sûr, la question ne peut être véritablement tranchée et ce d'autant plus que l'enclos n'a pu être étudié dans sa globalité. Cependant, certaines pièces (le glaive, un des quatre umbones, voire la pointe de lance), appartenant à l'équipement d'un légionnaire romain, ont très probablement été récupérées sur un ennemi. Quant à la louche, elle évoque des exemples mis au jour dans des camps du limes (Feugère, 1986). Tout comme la pelle à feu, elle peut faire partie du paquetage d'un militaire romain (impedimenta). Par ailleurs, le regroupement de deux umbones est comparable à ce qui a pu être observé à Corent et sur certaines représentations antiques. Enfin, les traces de décapitation mises en évidence sur l'un des crânes plaident en faveur d'un acte violent, commis sans véritable considération pour le sujet. Dès lors, l'ensemble suggère une prise de guerre faite après la bataille davantage que la vénération des reliques d'un ancêtre (Ricard, Gandia, 2012). Il est d'ailleurs possible que les crânes «familiaux » aient été récupérés après putréfaction des chairs, sans nécessité d'une découpe du sujet. Ces arguments, certes fragiles, laissent penser qu'on est bien là en présence d'un trophée, et permettent d'en proposer une restitution.

L'histoire est écrite par les vainqueurs, et elle n'a retenu que les victoires décisives des Romains. Les trophées mis au jour dans le Rhône et le Puyde-Dôme démontrent que la conquête ne s'est pourtant pas faite sans quelques défaites, épisodes moins glorieux qu'on ne jugea pas bon de relater.

\section{Références bibliographiques}

Arcelin P., Plana-Mallart R., 2011, « Expression monumentale des rites protohistorique en Gaule méditerranéenne et dans la partie nord-est de la Péninsule Ibérique. Stèles, bâtiments cultuels et sculptures ", in Roure R. et Pernet L. (éd.), Des rites et des Hommes : les pratiques symboliques des Celtes, des Ibères et des Grecs en Provence, en Languedoc et en Catalogne, Paris, Éd. Errance, p. 25-62.

BrunAUX J.-L., 2012, « Du prix et des usages de la tête Les données historiques sur la prise du crâne en Gaule », in Boulestin B., Henry-Gambier D. (dir.), Crânes trophées, crânes d'ancêtres et autres pratiques autour de la tête: problèmes d'interprétation en archéologie, Actes de la table ronde pluridisciplinaire (musée national de Préhistoire, Les Eyzies-de-Tayac, 14-16 octobre 2010), Oxford, Archaeopress (BAR International Series, 2415), p. 107-116

Brunaux J.-L., Rapin A., 1988, Gournay II : boucliers, lances, dépôts et trophées, Paris, Éd. Errance ( $\mathrm{n}^{\circ}$ special de Revue archéologique de Picardie), $245 \mathrm{p}$.

Ciesielski E., Duday H., Girard B., Roure R. MARTIN A., Agusti B., 2011, «La pratique des têtes coupées et les dépôts d'armes en Gaule méditerranéenne et dans le nord-est de la Péninsule Ibérique », in Roure R. et Pernet L. (éd.), Des rites et des Hommes : les pratiques symboliques des Celtes, des Ibères et des Grecs en Provence, en Languedoc et en Catalogne, Paris, Éd. Errance, p. 113-145.
DePEYRot G., 2008, Légions romaines en campagne: la colonne Trajane, Paris, Éd. Errance, 247 p.

Ferber E., Bellon C. Bouvier A., Motte S., 2012, Décines-Charpieu (Rhône). Le Montout - OL Land - tranche 1, rapport d'opération, Inrap-SRA RhôneAlpes, 6 vol. $(299,281,248,199,164,624$ p.).

Ferber E., Plantevin C., 2013, « Le Grand stade de Lyon, 6600 ans sous la pelouse », Archéologia, $\mathrm{n}^{\circ} 511$, juin 2013 , p. 34-43.

FeUGÈre M., 1986, « Note sur un type particulier d'instrument culinaire composite à l'époque romaine ", Germania, 62, p. 173-179.

Garcia M., Demierre M., 2011, « Le trophée de Corent (Puy-de-Dôme) ", in Les Gaulois. La fin d'un mythe Les Dossiers d'archéologie, hors-série ${ }^{\circ} 21$, octobre 2011, p. 52-53.

Guichon R, Poux M., 2011, "Le sanctuaire », in Poux M. (dir.), Corent, Voyage au coeur d'une ville gauloise, Paris, Errance, p. 141-175.

MANiQUeT C., 2008, « Le dépôt cultuel du sanctuaire gaulois de Tintignac à Naves », Gallia, 65, p. 273-326.

MANIQUET C., 2011, « Le sanctuaire gaulois de Tintignac (Corrèze), entre rituel guerrier et religion », in Les Gaulois. La fin d'un mythe, Les Dossiers d'archéologie, hors-série $n^{\circ} 21$, octobre 2011, p. 8-13.

NICOLAI C. vON, 2009, « La question des Viereckschanzen d'Allemagne du Sud revisitée», in BERTRAND I., Duval A. GoMez De Soto J., MAguer P. (dir.), Habitats et paysages ruraux en Gaule et regards sur d'autres régions du monde celtique, Actes du $\mathrm{XXXI}^{e}$ colloque international de l'Association française pour l'étude de lâge du fer (Chauvigny, 17-20 mai 2007), Chauvigny, Association des publications chauvinoises, p. 245-280.

Poux M. (dir.), 2008, Sur les traces de César, militaria tardo-républicaine en contexte gaulois, Actes de la table ronde (Glux-en-Glenne, 17 octobre 2002), Glux-en-Glenne, Bibracte, Centre archéologique européen (Bibracte 14), $463 \mathrm{p}$.

RiCARD J., GANDIA D., 2012, « Comment les Gaulois prélevaient-ils la tête de leurs ennemis ? Analyse des vertèbres cervicales découvertes sur le site de Ribemont-sur-Ancre (Somme) », Gallia, 69.2, p. 55-78.

RousSEAU É., 2012, « Pratique des têtes coupées chez les Gaulois : les données archéologiques », in Boulestin B., Henry-Gambier D. (dir.), Crânes trophées, crânes d'ancêtres et autres pratiques autour de la tête : problèmes d'interprétation en archéologie, Actes de la table ronde pluridisciplinaire (musée national de Préhistoire, Les Eyzies-de-Tayac, 14-16 octobre 2010), Oxford, Archaeopress (BAR International Series, 2415), p. $117-138$.

Testart A., 2012, « Pourquoi couper des têtes ?», in Boulestin B., Henry-Gambier D. (dir.), Crânes trophées, crânes d'ancêtres et autres pratiques autour de la tête: problèmes d'interprétation en archéologie, Actes de la table ronde pluridisciplinaire (musée national de Préhistoire, Les Eyzies-de-Tayac, 14-16 octobre 2010), Oxford, Archaeopress (BAR International Series, 2415), p. 29-30.

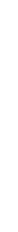

